\title{
Die „Seeräuberei der Barbareskenstaaten“ im Lichte des europäischen und islamischen Völkerrechts ${ }^{1}$
}

\author{
Von Almut Hinz, Leipzig
}

\section{Einleitung}

Als "Barbareskenstaaten" ${ }^{2}$ werden gewöhnlich die nordafrikanischen Regentschaften Tripolis, Tunis und Algier bezeichnet, mitunter wird auch Marokko dazugezählt. Sie bestanden etwa von 1500 bis 1830 und gehörten de iure zum Osmanischen Reich. De facto jedoch waren sie unabhängige kleine Reiche mit gewählten Herrschern. ${ }^{3}$ Ihre kontinuierliche Bedrohung der Handelswege in die Levante und nach Indien zog sie in den Kampf um Einfluss über den Handel zwischen den großen Seemächten. Das Ziel der Barbareskenstaaten war es dabei vornehmlich, Schiffe und Waren zu erbeuten sowie christliche Gefangene zu machen, die im Gegensatz zu Muslimen als Sklaven verkauft werden konnten.

Überarbeitete und um den wissenschaftlichen Apparat erweiterte Fassung des gleichnamigen Vortrages, den die Verf. am 20. September 2004 auf dem 29. Deutschen Orientalistentag in Halle/S. gehalten hat.

Die Wiedergabe arabischer Termini in lateinischer Schrift erfolgt nach den Regeln der Deutschen Morgenländischen Gesellschaft: C. Brockelmann, Die Transliteration der arabischen Schrift und ihre Anwendung auf die Hauptliteratursprachen der islamischen Welt, 1935.

Der Begriff "barbaresk" kann in Zusammenhang stehen mit dem Wort "Barbar", dessen Bedeutung jedoch nicht geklärt ist. Es handelt sich möglicherweise um eine Ableitung von den ältesten bekannten Bewohnern Nordafrikas, den Berbern. Es ist jedoch auch eine Herleitung vom Worte "barbar" nicht auszuschließen: ó $\beta \alpha \rho \beta \alpha \rho o$ ọ bezeichnet den Barbar, den Nichtgriechen und damit Ausländer sowie den der griechischen Kultur Unteilhaftigen - denn den Europäern erschienen sie als Inbegriff der ungesitteten Menschen. Vgl. A. Hinz, a.a.O., S. 122, Fn. 344.

$S$. Lane-Poole verweist auf das komplizierte Beziehungsgeflecht zwischen den regierenden Paschas, Deys, Beys bzw. Scherifen der Barbareskenstaaten und den europäischen Mächten und nennt diese noch ausstehende Darstellung eine "... task at once difficult and wearisom." (Ders., The Barbary Corsairs, 1896, S. 261).

4 Vgl. S. Dearden, A Nest of Corsairs: The Fighting Karamanlis of Tripoli, 1976, S. XIII, S. 15 f. Eine anschauliche Beschreibung über das Leben als Galeerensklave - aber auch über die Macht mitunter der Sklaven über ihre Herren - findet sich bei S. Lane-Poole, a.a.O., S. 214 f. bzw. S. $243 \mathrm{f}$. Zu den Zielen der Korsaren s. auch den Beitrag von D. Panzac, La course est-elle rentable? Le cas des régences barbaresques vers 1800, S. 201-213, in: Méditerranée, Moyen-Orient: Deux siècles de relations internationales. Recherches en hommage à Jacques Thobie, 2003, S. 201-220. 
Die europäischen Staaten und später auch die USA setzten sich gegen diese unwillkommene Erschwernis des Handels zur Wehr. In Verträgen mit den Barbareskenstaaten wurde die ungehinderte Fahrt durch das Mittelmeer gegen die Zahlung von Tributen (meist Schiffe, Waffen, maritime Ausrüstungen) vereinbart. Schiffe, die bei Kontrollen nicht ein mit den Barbareskenstaaten geschlossenes Abkommen vorlegen konnten, wurden entschädigungslos konfisziert. Die Besatzung wurde versklavt oder aber als Geiseln genommen, die sich nur gegen hohe Lösegelder freikaufen lassen konnten.

Die sog. Barbaresken waren zwar keine Mitglieder der abendländisch-christlichen Völkerrechtsgemeinschaft, doch bestanden dessenungeachtet vielfältige Beziehungen zwischen den europäischen und den Barbareskenstaaten. Im folgenden sollen diese Beziehungen gemäß europäischem und islamischem Rechtsdenken rechtlich eingeordnet werden insbesondere hinsichtlich der Fragestellung, ob der weitverbreitete Vorwurf der Seeräuberei durch die Barbareskenstaaten unter juristischem Gesichtspunkt tatsächlich Berechtigung hat.

\section{Die "Seeräuberei der Barbareskenstaaten"}

Im Rahmen der Beurteilung der sog. Seeräuberei der Barbareskenstaaten ist wiederholt erörtert worden, ob auch staatlich sanktionierter Seeraub (Kaperei) dem Tatbestand der Piraterie zuzurechnen ist. Nicht nur in Abhängigkeit vom christlichen oder muslimischen Hintergrund fällt die Antwort auf diese Frage unterschiedlich aus. Zunächst ist deswegen der Begriff der Piraterie näher zu untersuchen, anschließend daran die Situation sowohl der europäischen als auch der nordafrikanischen Staaten im fraglichen Zeitraum. ${ }^{5}$

\section{Der Terminus Piraterie}

In den Anfängen der mediterranen Schiffahrt - und nur um diese soll es hier gehen - waren Seehandel, Seekrieg, Kaperei und Seeraub noch ungetrennt. Bei Homer gilt der Seeraub als durchaus angesehene Tätigkeit für den Lebensunterhalt. ${ }^{6}$ Den Beginn des Seeraubs setzt

Die Verf. stützt sich hierbei ausschließlich auf Sekundärquellen, da die vorhandenen Primärquellen in Form von offiziellen Chroniken (z.B. Tunesien), privaten Chroniken (z.B. Tripolis), Konsular-Korrespondenzen der nordafrikanischen Konsulate, sog. Schiffspässen sowie Registern der Gesundheitsbehörden europäischer Häfen insbesondere von D. Panzac, aber auch von anderen, bereits ausgewertet wurden. Allerdings ist die Informationslage in einigen Bereichen in Ermangelung von Quellen unvollständig geblieben. Zu dem System der Schiffspässe s. S. Dearden, a.a.O., S. $4,15 \mathrm{ff}$. Vgl. Homer, Odyssee, III 105 f.; IX 39-42, 252-55; XIV 199-359 (Ausgabe von W. Schadewaldt, 1999). 
der griechische Historiker Thukydides zeitgleich mit den Anfängen der griechischen Seefahrt an. ${ }^{7}$ Rasch wuchs sich der Seeraub zu einer derart großen Plage aus, dass er sogar zu den Hauptmotiven der Neuen Komödie und der Tod durch Seeräuber epigrammatisches Thema wurde. In frühgeschichtlicher Zeit schritt erstmals die kretische Marine gegen den Seeraub ein, im 7. Jh. vor Chr. dann die Marine Korinths. ${ }^{8}$ Mehrere Versuche wurden von Athen, in hellenistischer Zeit von Rhodos, anschließend von König Eumelos und den Ptolemäern unternommen, um den Seeraub zu unterbinden. Im Jahre 67 v. Chr. sicherte Pompeius $^{9}$ die Schiffahrt durch eine Großoperation, in der 846 Boote versenkt oder erbeutet wurden. Mit ausreichenden finanziellen Mitteln versehen leitete Pompeius Rüstung und Planung mit großer Umsicht. Nach drei Monaten - der Krieg wurde gleichzeitig an allen gefährdeten Küsten geführt - waren im Mittelmeerraum die Seeräuber unterworfen und der Schiffsverkehr verlief wieder ungehindert. In der römischen Kaiserzeit wachten stehende Flottenverbände erfolgreich über die pax maritima. Die byzantinische Marine behauptete sich ab dem 7. Jh. n. Chr. zunächst gegen arabische Seeräuber, unterlag dann aber schließlich Ende des 12. Jahrhunderts. 10

Der französische Historiker Fernand Braudel fasst die Situation mit den folgenden Worten zusammen:

"Die Piraterie im Mittelmeer ist so alt wie die Geschichte. [...]. Im übrigen sind im Mittelmeer die Worte Piraterie und Piraten kaum gebräuchlich, zumindest nichts [sic] vor Beginn des 17. Jahrhunderts; von Kaperei und Korsaren ist die Rede, und der juristisch nicht eindeutige Unterschied, der freilich am Problem nichts Grundsätzliches ändert, ist von großer Bedeutung."11

Nicht nur etymologisch, sondern auch inhaltlich sind die für Seeraub verwendeten Begriffe Kaperei / Kaperkrieg, Korsarentum, Freibeutertum und Seeräuberei / Piraterie deutlich voneinander abzugrenzen. Zwar werden für "Korsar" - aus dem italienischen corsaro / corsare übernommen - gleichbedeutend Pirat, Seeräuber und Freibeuter verwendet, doch ist es von der eigentlichen Kaper insofern abzugrenzen, als dort ohne Kaperbriefe Handelsschiffe erbeutet wurden:

"Die Kaperei ist erlaubter Krieg und wird dies durch eine förmliche Kriegserklärung oder durch Kaperbriefe, Pässe, Aufträge, Instruktionen ... So merkwürdig uns solche fünf Bänden, 1979, Bd.5, Sp.64 f. 74.
Vgl. Thukydides, 1, 5, 1 (Ausgabe von A. Horneffer, [1984]).

Vgl. ebd., 1,8,1-3, 1,13,5 und die Dissertation (Univ. Greifswald) von F. Becker, Der Seeraub im Mittelmeer in den ersten zwei Jahrhunderten nach Alexanders des Großen Tode, 1922.

Siehe M. Gelzer, Pompeius. Lebensbild eines Römers, 1984: V. Kap.: Der Seeräuberkrieg, S. 65-

Vgl. Lexikon der Alten Welt, 2001, Bd. 3, S. 2755, und Der Kleine Pauly. Lexikon der Antike in

F. Braudel, Das Mittelmeer und die mediterrane Welt in der Epoche Philipps II., 1990, Bd. II, S. 694. 
Feststellungen rückblickend erscheinen mögen, Kaperei hat »ihre Gesetze, ihre Regeln, ihre lebendigen Bräuche und Überlieferungen «.",

Die 1856 durch die Seerechtsdeklaration von Paris ${ }^{13}$ offiziell abgeschaffte Kaperei hingegen bedeutete das Aufbringen von feindlichen Handelsschiffen durch Privatpersonen aufgrund eines Kaperbriefes einer kriegführenden Macht. Von wenigen fachwissenschaftlichen Ausnahmen abgesehen werden in der Literatur die hier genannten Begriffe in der Regel synonym verwendet. Zwei Autoren von Standardwerken, Dearden ${ }^{14}$ und Panzac ${ }^{15}$, gebrauchen bei dem von seiten der Barbareskenstaaten ausgehenden Seeraub den Begriff "Korsar", der im folgenden auch hier Anwendung findet. ${ }^{16}$

Rechtlich gesehen konnten die angreifenden Korsaren demzufolge keine Piraten ${ }^{17}$ sein, da sie von ihren Regierungen ausgestellte Briefe mit sich führten, die ihr Tun legitimierten. Genau besehen handelte es sich somit bei den Korsaren um legitimierte Mittelmeer-Seeräuber, die sich vor allem durch sorgfältig geplante und ausgeführte Unternehmungen sowie einen abgesprochenen Schlüssel ${ }^{18}$ von Anteil und Profit auszeichneten. ${ }^{19}$ Für den Antipiratischen Verein Hamburg hingegen stellte 1819 die "Seeräuberei der Barbaresken" kein kriegsrechtlich-strafrechtliches, sondern ein kriminelles Verhältnis zu Europa dar, da alle

Ebd.

Heute gilt für die meisten Staaten das Seerechtsübereinkommen der Vereinten Nationen von 1982. Mit Piraterie sind die Artikel 100-107 befasst, zentral ist Art. 101. Siehe z.B. The Law of the Sea. Official Texts of the United Nations Convention on the Law of the Sea of 10 December 1982, 1997.

S. Dearden, a.a.O.

D. Panzac, Barbary Corsairs. The End of a Legend 1800 - 1820, 2005.

Großen Einfluss auf die europäische Geschichtsschreibung hatten auch die folgenden Bücher von Salvatore Bono: I Corsari barbareschi (1964) und Corsari nel Mediterraneo, Christiani e musulmani fra guerra, schiavitù e commercio (1993). Die bis dahin ausschließlich auf den eigenen Quellen basierende europäische Geschichtsschreibung über die Barbareskenstaaten mußte relativiert werden, denn: "This one-sided European point-of-view has persisted for a long time and numerous are the books in which terrible North African pirates are set against heroic European sailors, defenders of the Cross against the Crescent." (D. Panzac, Barbary Corsairs. The End of a Legend 1800 - 1820, 2005, S. 1 f.).

Das lateinische Wort pirata für "Seeräuber, Pirat" stammt aus dem Griechischen und ist als

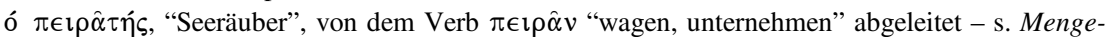
Güthling, Enzyklopädisches Wörterbuch der lateinischen und deutschen Sprache. Erster Teil: Lateinisch-Deutsch, 1954, S. 572, und W. Gemoll, Griechisch-deutsches Schul- und Handwörterbuch, ${ }^{5} 1954$, S. 587.

Für Details s. F. Braudel, The Mediterranean and the Mediterranean World in the Age of Philip II, 1972, S. 627 f., S. Dearden, a.a.O., S. XIII, S. 16, und den Beitrag von D. Panzac, La course estelle rentable? Le cas des régences barbaresques vers 1800, S. 213-220, in: Méditerranée, MoyenOrient: Deux siècles de relations internationales. Recherches en hommage à Jacques Thobie, 2003, S. 201-220. 
Staaten für freie Schiffahrt Tribut zahlen mussen. ${ }^{20}$ In der merkantilistisch-geschichtlichen Darstellung der Barbareskenstaaten bei Tönnies heißt es dementsprechend: “ $\mathrm{Zu}$ den empfindlichen Uebeln, welche [...] alle Seestädte Nord-Deutschlands [...] drücken, gehört die Sperrung der Schiffahrt nach dem mittelländischen Meere [...] wegen Unfriedens, welcher zwischen den Nord-Deutschen Staaten und den Barbaresken noch immer besteht. Nur die langwierige Gewohnheit hat dieses Uebel in der letzten Zeit weniger fühlbar gemacht, während es in seinen Wirkungen den Handel auf das schmerzlichste benachteiligt.,"21

\section{Die Lage der Barbareskenstaaten}

Vom 16. Jahrhundert bis in die ersten Jahrzehnte des 19. Jahrhunderts verbreiteten die Korsaren der Barbareskenstaaten Angst und Schrecken im Mittelmeer und stellten insbesondere für die europäischen Seefahrernationen ein großes Ärgernis dar. In ihren eigenen Ländern hingegen wurde ihnen Bewunderung zuteil, galten sie doch gewissermaßen als "Speerspitze des Islams" in der immerwährenden Auseinandersetzung mit den Nichtmuslimen. $^{22}$

Die Korsaren waren nicht ausschließlich Muslime: "Privateering often had little to do with either country or faith, but was merely a means of making a living. If the corsairs came home empty-handed there would be a famine in Algiers. Privateers in these circumstances took no heed of persons, nationalities or creeds, but became mere sea-robbers."23

Insbesondere seit dem Ende der Kriege gegen Spanien 1595 traten arbeitslose englische und holländische Atlantikkapitäne christlichen Glaubens in die Dienste der mehrheitlich muslimischen Korsaren. Die unterschiedliche Konfession war dabei von keiner Bedeutung. Ihre besser ausgerüsteten Schiffe machten diese weniger abhängig von Wind, Wetter und geographischen Gegebenheiten. ${ }^{24}$ Insgesamt aber war die Anzahl der christlichen Korsaren im Mittelmeer deutlich geringer als die der muslimischen. ${ }^{25}$

Vgl. H. Leip, Die Geschichte der Piraterie. 3000 Jahre Freibeutertum, 2002, S. 12.

P.D.W. Tönnies, Merkantilistisch-geschichtliche Darstellung der Barbaresken-Staaten und ihrer Verhältnisse zu den Europäischen und Vereinigten Nord-Americanischen Staaten, 1826, S. VIII.

22 Vgl. D. Panzac, Barbary Corsairs. The End of a Legend 1800-1820, 2005, S. 1. Vgl. S. v. Sivers, Nordafrika in der Neuzeit, S. 521, in: U. Haarmann, Geschichte der arabischen Welt, 2001, S. 502-604.

25 Vgl. F. Braudel, The Mediterranean and the Mediterranean World in the Age of Philip II, 1972, S. 633. 
Ein wichtiges Charakteristikum der Regentschaften war die weitgehende Autonomie, die sie seit dem 17. Jahrhundert in ihrer Beziehung zum Osmanischen Reich auszeichnete und sich in Form von diplomatischen Vertretungen ${ }^{26}$ und der Errichtung einheimischer Dynastien (insbesondere in Tunis und Tripolis) äußerte. Im 18. Jahrhundert nahmen, einhergehend mit den wachsenden Handelsbeziehungen zu den europäischen Staaten, die Kaperaktivitäten ab. Das Jahr 1793 stellte allerdings eine Wende dar: Mit den infolge der Französischen Revolution ausgelösten Kriegen kam es zu einer Wiederbelebung der Kaperei durch die Barbareskenstaaten bis etwa 1806. Ohne die Intervention europäischer Mächte erfolgte in diesem Jahr aus eigenem Antrieb eine Verlagerung von Kaperfahrten auf die Schaffung einer Handelsflotte in Anlehnung an das griechisch-osmanische Vorbild. ${ }^{27}$ Da die ehemaligen Korsaren jedoch nicht den Europäern gewachsen waren, kehrten sie zu ihrem Leben als Korsaren ${ }^{28}$ zurück - jedoch, wie Panzac schreibt, einmal zu viel: "It was once too many: and, unlike the seventeenth and eighteenth centuries and the tacit pact between both sides of the Mediterranean; there were no pacts, all to the advantage of Europe." 29 Aufgrund der wirtschaftlichen Überlegenheit und des Zusammenhalts Europas gegen die Barbareskenstaaten wurden diesen ihre Einnahmequellen entzogen. Darüber hinaus schwer getroffen von Naturkatastrophen und internen Machtkämpfen waren die nun schwach gewordenen Regentschaften Europa ausgeliefert. ${ }^{30}$

\section{Die Situation in Europa}

Ab dem 15. Jahrhundert wurden die nordafrikanischen Küstengewässer zunehmend von Korsaren beherrscht: Die Inseln Sizilien und Malta lagen in Segelweite, und die Korsarenschiffe mit nur geringem Tiefgang und großem Fassungsvermögen zum Abtransport der Beute eigneten sich vorzüglich für diese Gewässer.

Bis zum Ende des 15. Jahrhunderts hatte das Osmanische Reich seine Interessen bis ins westliche Mittelmeer ausgedehnt, und als die Granadiner den osmanischen Sultan 1487 um Hilfe im Kampf gegen Kastilien ersuchten, schickte er ihnen Korsaren. Doch auch diese

Panzac schreibt dazu: "Real diplomatic relations between the regencies and Europe did not officially begin until the seventeenth century, and were at one and the same time created and conditioned by the practice of privateering." D. Panzac, Barbary Corsairs. The End of a Legend $1800-$ 1820,2005 , S. 25.

27 Vgl. ebd., S. 3 ff.

28 Für eine detaillierte Beschreibung über die Schiffe sowie die Besatzung der Korsaren s. $D$. Panzac, Barbary Corsairs. The End of a Legend 1800 - 1820, 2005, S. 45-56 bzw. S. 56-72.

29 Ebd., S. 4.

30 Vgl. ebd., S. 4 f.; verursacht durch Pest und Hungersnöte schrumpfte die Bevölkerung sehr rasch, s. S. 303-309; für interne Machtkämpfe - hier am Beispiel von Tripolis - s. S. 298 f. 
vermochten nicht den Fall Granadas zu verhindern und wurden 1495 zurückbeordert. Im Namen ihres Sultans unternahmen Korsaren ab Anfang des 16. Jahrhunderts erste Schritte zur Rückeroberung des islamischen Westens. In den Jahren 1512/13 setzten sie sich in Tunis, 1516 in Algier fest. In den Folgejahrzehnten waren beide Parteien abwechselnd siegreich: 1571 unterlagen die Osmanen bei Lepanto im östlichen Mittelmeer gegen die vom Vatikan und Venedig ausgerüstete Flotte, und zwei Jahre später gelang es den Spaniern, die Korsaren - allerdings nur für ein Jahr - aus Tunis zu vertreiben. Im 17. und 18. Jahrhundert geriet der Kleinkrieg, der zwischen muslimischen und christlichen Korsaren gegeneinander und um die jeweils gegnerischen Handelsschiffe geführt wurde, eher zu einem Nebenschauplatz: in Nordafrika kämpften Korsaren im Namen des vom Osmanischen Reich repräsentierten Islams gegen christliche Korsaren weiter, die von Frankreich direkt oder indirekt über Malta unterstützt wurden, wo der Johanniter- / Malteserorden die christliche Korsarenflotte befehligte. Dessenungeachtet war dieser Seekrieg eine große Belastung für den Handel und die Küstenbewohner des nördlichen Mittelmeerraumes. ${ }^{31}$ Über die kriegerischen Handlungen im Dienste des osmanischen Sultans hinaus griffen die muslimischen Korsaren vor allem unbewaffnete Handelsschiffe auf dem Weg in die Levante an, da sie sich persönlich als im Krieg befindlich mit jeder Nation sahen, mit der sie keinen Friedensvertrag oder finanzielle Abkommen hatten. ${ }^{32}$ Im Jahre 1655 errang der englische Admiral Robert Blake einen Sieg gegen die Korsaren, die bis dahin englische und andere Handelsschiffe behindert hatten. 1678 kam es unter anderem zu einem Friedensund Handelsvertrag zwischen England und Tripolis, wo ein englisches Konsulat und bis 1783 sieben Konsulate weiterer Mächte eingerichtet wurden. ${ }^{33}$ Ihre Aufgabe bestand zuvörderst in der Förderung des Handels: Sie hatten die freie Passage für Handelsschiffe zu sichern, für die Freilassung vertragswidrig gefangener Schiffsangehöriger zu sorgen und sich um die Sklaven und Schutzbefohlenen anderer Staaten ohne konsularische Vertretung vor Ort zu kümmern. Internationale Abkommen sicherten diese Schutzfunktion der mit weitreichenden Vollmachten ausgestatteten Konsuln. ${ }^{34}$ So war der Status der Europäer im Osmanischen Reich in den "Kapitulationen”35 von 1761 festgeschrieben: Die Angehörigen

31

32

33

34

35

Vgl. S. v. Sivers, a.a.O., S. 507-520.

Vgl. S. Dearden, a.a.O., S. 4, 15 f.; siehe auch die folgenden Fn. 33 und 34.

Zu einem Rückgang der seeräuberischen Aktivitäten kam es zeitweilig, als du Quesne 1681 und d'Estrées 1685 eine größere Anzahl von Korsarenschiffen zerstörten und auch, weil keine Hungersnot die Barbaresken zur Seeräuberei trieb. Vgl. P.D.W. Tönnies, a.a.O., S. 69.

$\mathrm{Zu}$ Einzelheiten s. J. Mössner, Die Völkerrechtspersönlichkeit und die Völkerrechtspraxis der Barbareskenstaaten, 1968, S. 123-129: Übersicht über den Inhalt der Kapitulationen und die Aufgaben der Diplomaten.

Über die Bedeutung als einseitige Unterwerfungserklärung hinaus bezeichnet Kapitulation auch (von lat. capitulare = in Kapitel einteilen) einen Vertrag im allgemeinen. Bis 1856 stand die Türkei wegen "abweichender religiöser Anschauungen" und wegen "wesensfremder Kultur" außerhalb der westlichen Völkerrechtsgemeinschaft. Die sog. Kapitulationen können als reziproke, vollgültige rechtliche Verträge angesehen werden, die keine enge Verbindung zu dem Recht des 
der Vertragsstaaten waren von der Rechtsprechung des Paschas ausgenommen und unterlagen statt dessen in Strafsachen und zivilrechtlichen Streitigkeiten mit Landesbewohnern der ausschließlichen Gerichtsbarkeit eines bei dem Konsulat eingerichteten Gerichtes. ${ }^{36}$ Für die europäischen Staatsangehörigen war die Stellung der Konsuln ${ }^{37}$ somit von herausragender Bedeutung, da diese - häufig durch finanzielle Zuwendungen - durchaus über Einfluss beim Pascha verfügten. Meist hatte ein Pascha jedoch auch keine Skrupel, Abmachungen in seinem Sinne zu brechen, sah er es wegen der politischen Situation als gerechtfertigt an oder ließ es seine Finanzlage opportun erscheinen. Es war durchaus eine taktische Frage, die bestehenden Verträge zu brechen, um in neuen Verhandlungen höhere Tribute festsetzen zu können. ${ }^{38}$

Kleinere Staaten wie Venedig, Genua, Malta, Sizilien, der Vatikan und Spanien, die sich kein Friedensabkommen erkaufen konnten oder wollten, hatten das Nachsehen. ${ }^{39}$ Die Seemächte England, Frankreich und Niederlande hätten sich leicht zusammenschließen und den Umtrieben der Korsaren ein Ende bereiten können. Das lag jedoch aus Gründen des Wettbewerbs nicht im Interesse dieser drei Seefahrerstaaten, so dass alle maritim interessierten Staaten mehr oder weniger bereitwillig ihre Tribute zahlten. ${ }^{40}$

Eine Veränderung dieser Situation trat nicht mit den durch die Französische Revolution bedingten Umbrüchen ein - die Wirtschaftsaktivitäten und -beziehungen Frankreichs mit dem Maghreb waren davon unberührt -, sondern bereits durch die Störung des Gleichgewichts im Mittelmeer, die durch die Kriegserklärung Englands gegen Frankreich von 1783 einsetzte. Die französische Flotte erwies sich als der englischen unterlegen und war im Mittelmeerraum kaum mehr anzutreffen. Darüber hinaus wirkte sich Napoleons Vorrücken in Italien 1796 störend auf die Beziehungen zwischen dem Maghreb und Genua aus. Auch gelangten durch die in der Kontinuität der durch die Französische Revolution hervorgerufenen Konflikte stehenden Koalitionskriege in Europa (1792-1815) die vertraglich den Barbareskenstaaten zugesicherten Bezahlungen der Europäer nur noch unregelmäßig zu

Dschihad aufwiesen und z. T. auch in Widerspruch zum islamischen Recht standen (z.B. Vertragsdauer). Vgl. dazu J. Mössner, a.a.O., S. 82 f., 147 f. Auf S. 89 (ebd.) findet sich ein Verzeichnis aller Verträge mit ihren Fundstellen.

40

Vgl. J. Mössner, a.a.O., S. 129-131.

Vgl. F. Rodd, General William Eaton. The Failure of an Idea, 1932, S. 56 f.

Vgl. M. Tully, Letters written during a ten years' Residence at the Court of Tripoli, 1957, S. 8-11.

Für ein tabellarisches Verzeichnis der Seehäfen, nach und von welchen die Schiffahrt von nicht mit den Barbareskenstaaten durch Abkommen verbundenen Staaten unter fremden Flaggen hatte betrieben werden müssen, vgl. P.D.W. Tönnies, a.a.O., S. 215-226. Keine Verträge mit den Barbaresken geschlossen hatten z.B. Preußen, Mecklenburg, Oldenburg, Lübeck, Bremen und Hamburg, siehe ders., S. 181.

Vgl. S. Dearden, a.a.O., S. 15, auch S. 24: So lag es beispielsweise im Interesse Frankreichs, die Aktivitäten der Barbareskenstaaten zwar zu kontrollieren, nicht aber zu beenden. 
den Adressaten. Die abnehmenden Einkünfte, aber auch der Drang nach Geltung sowie die diplomatisch ungesicherte Lage, förderten eine Rückkehr der Kapertätigkeiten der Barbareskenstaaten. Napoleons Ägpytenfeldzug 1798 wirkte sich dabei zunächst für die Barbaresken positiv aus, da Napoleon den Malteserorden, einen wichtigen Gegenspieler der Barbareskenstaaten, entscheidend schwächte. Mit der Eroberung Ägyptens durch Frankreich wurde schließlich der seit 250 Jahren währende Frieden zwischen dem Osmanischen Reich und Frankreich beendet, und der osmanische Sultan besann sich auf die ihm formell unterstehenden nordafrikanischen Regentschaften und zwang diese, gegen Frankreich Krieg zu führen. ${ }^{41}$ Allerdings "[...] verstand [es Napoleon,] die Mittel und Wege auszufinden, die früheren ungestörten Friedens-Verhältnisse wieder herbei zu führen, und das Geschehene zu entschuldigen und zu beschönigen."42 Mit Napoleons Dekreten aus Berlin (1806) und Mailand (1807) wurde im Zeitraum 1807-14 jedoch alle Schiffahrt mit und zu den Barbaresken-Häfen eingestellt. 1814 forderten die Barbaresken die ausstehenden Tributzahlungen dieser Jahre, bevor ehemalige "Freundschaftsverhältnisse" wieder eingerichtet werden könnten. Es folgten neue Arrangements, womit sich die Betroffenen bewusst für die Tributzahlungen entschieden und somit gegen die aktive Bekämpfung der Barbaresken. Eine Initiative im Rahmen des Wiener Kongresses, England mit der Bekämpfung der Barbaresken zu beauftragen, verlief ergebnislos. ${ }^{43}$

Die Korsaren stellten etwa 300 Jahre für Europa eine Störung und ein Ärgernis dar, doch hatten sie keinen nachhaltigen Einfluss auf das europäische Leben oder die Kultur gewinnen können. Das drückt sich auch in der dürftigen Literaturlage aus. Die Barbareskenstaaten waren als entfernt gelegene, kleine Dependencen des Osmanischen Reiches für Europa ohne größere Bedeutung und hatten lediglich solange Bestand, wie Europa mit internen Auseinandersetzungen (16.-18. Jahrhundert) befasst war. ${ }^{44}$

4. Die USA und die Regentschaft Tripolis

Seit dem Bestehen der USA und dem durch den US-amerikanischen-britischen Gegensatz verursachten Wegfall des Schutzes durch die englische Flotte waren US-amerikanische Handelsschiffe an Auseinandersetzungen mit den Barbareskenstaaten beteiligt, da der Umfang des Seehandels in Übersee (Westindische Inseln, Europa) Tributzahlungen der Vereinigten Staaten an die Barbareskenstaaten in keiner Weise rechtfertigte. ${ }^{45}$ Unter USPräsident Jefferson wurde der Vorschlag gemacht, mit den anderen betroffenen Ländern

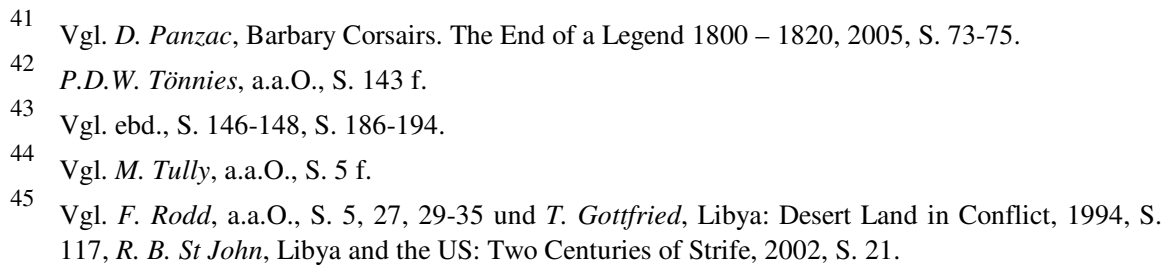


eine Flotte zusammenzustellen und die Barbareskenstaaten auf diese Weise unter Blockade zu stellen. Aus finanziellen Gründen mussten die USA von ihrem Vorschlag ablassen, und die europäischen Länder sahen keine Veranlassung dafür, ein Vorhaben auszuführen, das insbesondere den USA genutzt hätte. Somit schlossen sich die US-Handelsschiffe meist englischen, niederländischen, portugiesischen oder spanischen Konvois an und waren dadurch vor den Korsaren geschützt. Aufgrund einer Ermächtigung des US-Kongresses war es Politikern wie Jefferson, Franklin und Adams möglich, Verträge für die USA mit den Barbareskenstaaten abzuschließen. ${ }^{46}$ Gleichzeitig wurden in den USA auch militärische Maßnahmen erwogen, denn es war wiederholt zu Zwischenfällen mit den Korsaren gekommen. Andererseits scheute man jedoch militärische Maßnahmen. Selbst als Tripolis den USA bereits - berechtigterweise ${ }^{47}$ - den Krieg erklärt hatte, lehnte es Präsident Jefferson mit Verweis auf die US-Verfassung ab, Tripolis im Gegenzug den Krieg zu erklären. ${ }^{48}$ Drei Monate nach der tripolitanischen Kriegserklärung entstand erstmals ein konkreter Aktionsplan der Amerikaner, nachdem erneut einige ihrer Staatsbürger durch Korsaren ums Leben gekommen waren: Tripolis sollte durch eine Blockade ausgehungert werden. Die Aussicht auf Erfolg schien nicht gering, da Tripolis stark von Lebensmitteleinfuhren über See abhängig war. Allerdings wurden von den Amerikanern einige geographische Faktoren unterschätzt: Ohne verlässliche Seekarten oder die entsprechenden Kenntnisse war die nordafrikanische Küste wegen der dort typischen, unberechenbaren Windverhältnisse für Blockadeschiffe im Segelbetrieb kaum ohne Risiko zu befahren. ${ }^{49}$ Auch hatten die Amerikaner die schwierige Nachschublage unzureichend berücksichtigt, die bei einem Monat Segelzeit ohne Stützpunkte in der Region auftreten mussten. Die Ernsthaftigkeit der USKriegsführung wurde von den Korsarenführern ohnehin nur als gering eingeschätzt, da die Amerikaner ihre Gefangenen nicht wie die Korsaren versklavten, sondern bei Kapitulation freiließen, ein Verhalten, das als Schwäche ausgelegt wurde. Abgesehen vom unzulänglich kalkulierten Kräfteaufwand hätten die Vereinigten Staaten im Frühstadium ihrer Staatswerdung allerdings ohnehin nicht die Mittel besessen, eine größere und besser ausgerüstete

Vgl. die umfassende Dissertation von R. Irwin, The Diplomatic Relations of the United States with the Barbary Powers 1776-1816, 1931, S. 187 f. Die offiziellen Beziehungen zwischen den USA und Tripolis begannen mit einem Vertrag von 1796 - s. R. B. St John, The Terrorism Problem in Libyan-American Relations: Past and Future, in: Policy Briefs (Washington: The Middle East Institute), 30.4.2002, S. 19.

So kamen die Vereinigten Staaten ihren vertraglichen Pflichten in Hinblick auf finanzielle Zuwendungen und Geschenke nicht vollständig nach und behandelten vertragswidrig Tripolis nicht entsprechend den anderen Barbareskenstaaten - vgl. R. B. St John, Libya and the US: Two Centuries of Strife, 2002, S. 23.

Für Einzelheiten s. H. P. Nash, The Forgotten Wars. The Role of the U.S. Navy in the Quasi War with France and the Barbary Wars 1798-1805, 1968, S. 177-189 (9. Kapitel), und Band I der vom U.S. Office of Naval Records and Library herausgegebenen sechsbändigen Navel Documents Related to The United States Wars With The Barbary Powers, 1939-1944.

Vgl. C. Forester, The Barbary States, 1953, S. 16, 21 f., 35-47. 
Streitmacht zu entsenden. ${ }^{50}$ Der Krieg mit Tripolis zog sich daher unerwartet lang hin. ${ }^{51}$ Der Friedensvertrag, den 1805 die USA und der Pascha von Tripolis, Yusuf Qaramanli, aushandelten, war die Grundlage für ein pragmatisch bestimmtes Auskommen. ${ }^{52}$ Dabei war Grund für das Einlenken des Paschas weniger ein militärischer Erfolg der Amerikaner als vielmehr ein drohender Aufstand in Tripolis. ${ }^{53}$ Die sog. Korsarenzeit war mit der Entmachtung des Deys von Algier 1830 endgültig beendet. ${ }^{54}$

Abschließend ist festzuhalten: Die Vereinigten Staaten leisteten den Korsaren insbesondere durch den US-Tripolitanischen Krieg als erste - später dann gemeinsam mit England gezielten Widerstand gegen ihre Raubpraktiken, ohne wie die etablierten Seefahrernationen sich ihren Schutz quasi zu erkaufen. Damit ermutigten sie auch die anderen betroffenen Staaten zum Widerstand: "The successes of the English and American fleets had produced their effects, not so much in arresting the cours of piracy, as in encouraging the European States to defy the pirates." 55

Vgl. C. Forester, a.a.O., S. 47 f., 56 f., 153, u. S. Dearden, a.a.O., S. 151-155. Chronologisch geordnetes Quellenmaterial bieten die sechs umfangreichen Bände mit diplomatischer Korrespondenz und Marinequellen mit Bezug zu den mitunter kriegerischen Auseinandersetzungen zwischen den USA und den Barbareskenstaaten in der Zeit zwischen 1785 bis 1897: U.S. Office of Naval Records and Library, a.a.O. Für die zwischen den beiden Parteien ausgefochtenen Schlachten s. Takar, Ma ${ }^{\mathrm{c} a}$ rik Ṭarābulus baina al-ustūl al-lībī wa-'l-ustūl al-amrīkī fill-qarn at-tāsi ${ }^{\mathrm{c}}$ 'ašr, o.J. [Die Schlachten vor Tripolis zwischen der libyschen und der amerikanischen Flotte im 19. Jahrhundert].

51 Für Beschreibungen des Krieges s. z.B. J. Field, America and the Mediterranean World 19761882, 1969, S. 49-58 und J. L. Cathcart, Tripoli, First War with the United States. Inner History, 1901; S. Lane-Poole, a.a.O., S. 274-291, L. Rait, Al-Ḥamālāt al-amrīkīya calā šamāl Ifrīqiyā, o.J., [Die amerikanischen Angriffe auf Nordafrika] und $M .{ }^{c} U . a \check{s}-\check{S} a t \overline{1} w \overline{1}$, Harb al-qarșana baina duwal al-mağrib al-'arabī wa-'l-wilāyāt al-muttahida, 1993, [Der Piratenkrieg zwischen den Vereinigten Staaten und den Staaten des Maghreb].

Das Vertragswerk ist abgedruckt bei H. Miller, Treaties and Other International Acts of the United States of America, o.J., Bd. II, S. 520-556, auf Arabisch in der an der Qār-Yūnis-Universität in Benghasi angefertigten Magisterarbeit von ${ }^{c} A$. al-Kurğulī, Daur al- ${ }^{c} \bar{a} m i l$ al-īdūlūğì fil-- ${ }^{c}$ alāqāt allībīya al-amrīkīya (1970-1995), 1997, S. 235-240, [Die Rolle des ideologischen Faktors in den libysch-amerikanischen Beziehungen 1970-1995]. Nicht bekannt war dem US-Außenministerium ein geheimes Zusatzabkommen des Vertrages, das der US-Verfassung widersprach - Einzelheiten dazu bei F. Rodd, a.a.O., S. 263, 268 ff.

53 Vgl. F. Rodd, a.a.O., S. 266 f.; S. Dearden, a.a.O., S. 206-210. R. B. St John stellt in seinem Aufsatz The Terrorism Problem in Libyen-American Relations, 2002, S. 26 (in: Policy Briefs, Washington: The Middle East Institute, 30.04.2002) fest, dass das Ergebnis des Krieges durchaus positiv für die Qaramanlis ausfiel, hatten sie doch die USA geschlagen und somit ihr Ansehen als internationale Seemacht erhöht.

Siehe C. Lloyd, English Corsairs in the Barbary Coast, 1981, S. 165; R. W. Irwin, a.a.O., S. 192 ff., sowie das 5. und 6. Kapitel des 2. Teils bei E. Rossi, Storia di Tripoli e della Tripolitania dalla conquista araba al 1911, 1968, S. 221-238, und S. 239-258.

S. Lane-Poole, a.a.O., S. 301. Siehe auch ders. S. 292 f. 
Denn die Amerikaner waren "[...] die ersten, deren Kongress es weder respektabel, noch profitabel fand, weiteren Tribut zu zahlen, und so wurde denn zur Strafe für Gefangene[,] Piraterien und zum Zwecke der Befreiung von dem Tribut von 1804 [sic] die kleine amerikanische Flotte nach Afrika geschickt." ${ }^{, 56}$ Dazu kam es unmittelbar nach Beendigung des Krieges mit Großbritannien. 1815 sandten die USA eine Flotte nach Algier und erreichten bereits nach zweitägigen Verhandlungen - am 30. Juni 1815 - die Unterzeichnung eines Vertrages. Darin galten Geldzahlungen an die Korsaren als abgeschafft, auch mussten die Gefangenen und das konfiszierte fremde Eigentum zurückgegeben werden. Darüber hinaus wurde den USA vertraglich der Status einer "begünstigten Nation” zugesichert. 57

Ohne die Auseinandersetzungen mit den Barbareskenstaaten wäre es nicht relativ rasch nach der Staatsgründung zur Schaffung der US-Marine gekommen, wie Irwin schreibt: "[...] the Barbary involvements brought forth the United States Navy, an institution which was designed to protect lives and property of Americans irrespective of state or class, $[\ldots] ., 58$

\section{Das Völkerrecht nach islamischer Rechtsauffassung}

Trotz der engen Verknüpfung von Recht und Religion im Islam kann insgesamt dennoch von einer eigenständigen Rechtslehre gesprochen werden, da der Islam neben seinen religiösen und moralischen Aspekten auch ein Rechtssystem bietet. ${ }^{59}$ Die islamische Jurisprudenz geht dabei von religiösen Prinzipien aus, denen besonders im Bereich des Staates in der Realität kaum etwas je entsprochen hat. Die Vorschriften für das Verhalten der Muslime gegenüber der nichtmuslimischen, "ungläubigen" Außenwelt sind in der Theorie umfassend herausgearbeitet und abgehandelt worden. Dieser Teil des islamischen Rechts kann dabei nicht dem Begriff Völkerrecht entsprechen, wie er heute in der Regel verstanden wird. Denn die islamische Völkerrechtslehre umfasst nicht die Theorie des Rechtsverkehrs muslimischer Staatswesen untereinander oder das ius in bello der $\check{s} a r \bar{I}^{c} a$, dem religiös begründeten Recht des Islams. Sie hat statt dessen das Verhältnis des fiqh $^{60}$, der isla-

Vgl. S. Lane-Poole, a.a.O., S. 293. East, 1955, J. Schacht, An Introduction to Islamic Law, 1964, und I. Edge (Hg.), Islamic Law and Legal Theory, 1996.

Fiqh, eigentlich "Kenntnis, Jurisprudenz", umfasst die Lehre von den Rechtsquellen (wie Koran, Hadith) und die Lehre von den gesetzlichen Einzelbestimmungen. Dabei existiert kein einheitliches Gesetzeswerk, das für alle islamischen Richtungen verbindlich ist, sondern die Bestimmungen sind niedergelegt im Koran und in privaten Arbeiten islamischer Rechtsgelehrter. Vgl. aus- 
mischen Gesetzeswissenschaft, zu dem Normenkomplex zum Thema, der aufgrund gegenseitiger Anerkennung für die Beziehungen zwischen der islamischen Gemeinschaft und den nichtmuslimischen Gemeinschaften in Frage kommt. ${ }^{61}$

Der Völkerrechtler Kruse verwendet in seiner Dissertation über die islamische Völkerrechtslehre den Begriff Muslimisches Völkerrecht, das er als Verkehrsrecht muslimischer Staaten untereinander bezeichnet, und was in der islamischen Rechtslehre dem Staatsrecht zugeordnet wird. ${ }^{62}$ Das Islamische Völkerrecht hingegen definiert er im Gegenzug als "Vertragsvölkerrecht", es ist die Lehre vom Völkerrecht setzenden Staatsvertrag mit Nichtmuslimen. Eine besondere Bedeutung dabei haben die sog. siyar, "Verfahrenswei-

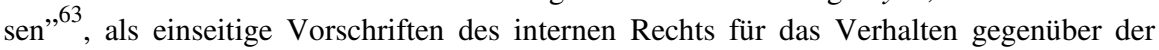
nichtmuslimischen Umwelt. Kruse nennt es auch "unilaterales Außenrecht". Aus den siyar und dem Vertragsvölkerrecht entwickelte sich nach Kruse das "partikuläre Völkergewohnheitsrecht" im Verkehr mit den nichtmuslimischen Staaten. Seine Formen wurden im wesentlichen von der Praxis bestimmt, waren aber durchaus auch beeinflusst von den jeweils geltenden völkerrechtlichen Normen. ${ }^{64}$

Im folgenden Abschnitt soll es insbesondere um den Dschihad und seine Suspendierung über das Fremden- und Vertragsrecht gehen.

führlich zu fiqh und šarĩ $a$ T. Nagel, Das islamische Recht, 2001, S. 3-10, H. Krüger, Fetwa und Siyar, 1978, S. 29, Fn. 37.

61 Vgl. H. Kruse, Islamische Völkerrechtslehre, 1953, S. 5 ff., M. Khadduri, International Law, in: M. Khadduri / H. Liebesny (Hg.), Law in the Middle East, 1955, S. 349-372, H. Krüger, a.a.O., S. 29, und L. Al-Zwaini / R. Peters, A Bibliography of Islamic Law, 1980-1993, S. 54-59 (International Law).

62

Vgl. dazu ausführlich J. Mössner, a.a.O., S. 64-70, H. Krüger, a.a.O., S. 34-36.

63

64

Vgl. H. Krüger, a.a.O., S. 31 f., 34.

Vgl. H. Kruse, a.a.O., S. 9, 12. 


\section{Die Stellung von Nichtmuslimen im sog. Islamischen Völkerrecht}

Das Islamische Völkerrecht enthält die Rechtsvorstellungen über das Verhältnis zwischen islamischen und nichtislamischen Staaten. Zentral ist hierbei Vers 5 der Koransure 9: “Tötet die Götzendiener, wo ihr sie auch finden mögt." Neben Sure 8, Vers 40, und Sure 2, Vers 194, bildet dieser Vers den Ausgangspunkt für die Pflichten der Muslime in ihrem Verhältnis zu den Ungläubigen (siyar), nämlich, diese zu überwinden. ${ }^{65}$ Dieses Verhältnis ist grundsätzlich von der Lehre des Dschihad geprägt. Während heutige islamische Gelehrte unter Dschihad eher den Kampf (wörtlich: die "Anstrengung”) gegen das Böse im Menschen verstanden wissen wollen, umfasst Dschihad klassischerweise durchaus die Überwindung der Ungläubigen, die außerhalb des islamischen Gebietes grundsätzlich als rechtlos gelten: "Nach allen Quellen des traditionellen islamischen Völkerrechts [...] trifft die Muslime die Rechtspflicht ("farḍ") den nichtislamischen Teil der Welt ("dār al-ḥarb") grundsätzlich durch "ǧihād" (Glaubenskrieg) "auf dem Wege Allahs" ("fī sabīl Allāh”) zu islamisieren."66

Von den vielen Einzelheiten des Rechtes des Dschihad ist auch auf die Form des Seekrieges zu verweisen, der von den Barbareskenstaaten ausschließlich gepflegt wurde. ${ }^{67}$ In der Praxis mischte sich die religiöse Begeisterung dabei durchaus mit dem materiellen Vorteil des Beutemachens.

Grundlegend für jede Suspendierung des Dschihads ist die Lehre vom amān, dessen historische Wurzel im altarabischen Gastrecht begründet ist. ${ }^{68}$ In der islamischen Gesetzeswissenschaft ist es zum Rechtsinstitut der Sicherheitszusage ausgeprägt worden. ${ }^{69}$ Mössner beschreibt die Wirkung von amān folgenderweise: "Ohne aman ist jeder Nicht-Muslim mit seinen Gütern fay (Beute), durch den aman wird er unantastbar (hisma) für die Geltungsdauer des aman.",70

Für eine Abmilderung dieser doktrinär-emotionalen Konfrontationshaltung erwies sich der folgende Grundgedanke als gangbarer Weg: Der amān kann auf dreierlei Weise erfolgen: 1. Juden und Christen genießen als Angehörige von "Buchreligionen" eine Sonderstellung

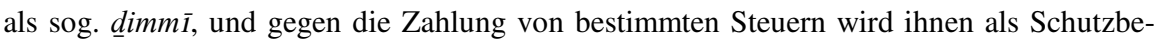

65

66

67

68

69

70

Vgl. H. Krüger, a.a.O., S. 154.

Ebd., S. 117. Ausführlicher: S. 118 f.

Vgl. H. Kruse, a.a.O., S. 71, 73.

Siehe W. Heffening, Beiträge zum Rechts- und Wirtschaftsleben des islamischen Orients, 1925, S. 9-14 (zur Terminologie) sowie S. 15-28 (der amān im fiqh).

Vgl. ausführlich dazu: W. Heffening, a.a.O., S. 28-37.

J. Mössner, a.a.O., S. 74. 
fohlenen Sicherheit innerhalb des islamischen Staatsverbandes gewährt. Sie gehören als Bürger mit gewissen Einschränkungen für immer zum "Gebiet des Islams" (dār al-islām). 2. Nichtmuslimischen Kaufleuten wird aus Gründen der persönlichen Sicherheit eine

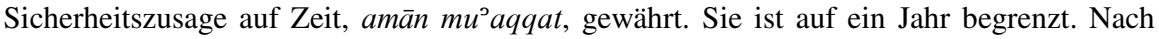
Verlauf eines Jahres erhält der Fremde, in diesem Zeitraum musta min genannt, ohne eigenes Zutun des Status des $\underline{d i m m}^{\mathrm{i}} \mathrm{i}$ und darf das islamische Gebiet nicht mehr verlassen. ${ }^{71}$ Drittens, und das ist an dieser Stelle relevant, können nicht nur einzelne Fremde, sondern auch nichtislamische Staaten Sicherheit vor den Angriffen von Muslimen erhalten. Der Dschihad kann nämlich suspendiert werden, wenn sein Ziel, dem Islam Vorteile zu verschaffen, nicht aussichtsreich ist. In diesem Fall wird ein Staatsvertrag, muwādac $a$, zwischen dem islamischen und dem nichtislamischen Gebiet geschlossen, durch den das nichtislamische Gebiet zu Vertragsland wird (dār aș-șulh $)^{72}$

Damit rückt Kruses Definition des Islamischen Völkerrechts in den Vordergrund: "Die Völkerrechtslehre der islamischen Jurisprudenz ist daher in den dogmatischen Erörterungen über den Abschluss von Verträgen zwischen der muslimischen Gemeinde und ungläubigen Staatswesen zu suchen. An dieser Stelle geben die unilateralen Normen für das Verhalten nach außen Raum für das bewusste und gewollte Zustandekommen mehrseitig verbindlicher Regeln. Darum ist aber auch die Völkerrechtslehre eingebettet in die siyar, die Gesamtheit der Vorschriften des fiqh für das Verhalten gegenüber den Ungläubigen, denn diese bestimmen, ob und wann ein solcher Vertrag rechtlich möglich ist und worauf er sich erstrecken kann."73

Im Zeitalter der europäischen Hegemonie dehnte sich das moderne Völkerrecht, das im Verkehr der christlich-abendländischen Staaten untereinander entstanden war, auf die nichtchristliche Welt aus. Seit der Mitte des 19. Jahrhunderts brachte die Rezeption dieses Rechtes durch die außereuropäischen Staaten andere zwischenstaatliche Rechtsordnungen zum Verschwinden. ${ }^{74}$ Hinsichtlich der "Seeräuberei" der Barbaresken ist dieser Umstand jedoch noch nicht relevant, so dass diese vor dem Hintergrund des Islamischen Völkerrechts gesehen werden muss.

Vgl. Sure 9, Vers 29, J. Mössner, a.a.O., S. 75, T. Nagel, a.a.O., S. 98 ff., und W. Heffening, a.a.O., S. $18 \mathrm{f}$.

72 Vgl. T. Nagel, a.a.O., S. 104. Zu ausführlichen Anmerkungen zum Staatsvertrag s. H. Krüger, a.a.O., S. 119-123.

H. Kruse, a.a.O., S. 8. Im Osmanischen Reich z.B. setzte sich der Şeyh ül-Islâm unter Anwendung der Rechtsinstitute "Notwendigkeit/Notstand" ("ḍaūra") und "öffentliches Interesse" ("mașlaḥa") wiederholt für die Suspendierung des Dschihads durch Staatsverträge ein, ohne allerdings dabei den Gedanken des kriegerischen Dschihads aufzugeben - vgl. H. Krüger, a.a.O., S. 154.

Vgl. H. Kruse, a.a.O., S. 1. 
2. “Anstrengungen im Dienste des Islams" und "Seeräuberei"

Im vorliegenden Fall steht die Begegnung der muslimischen Staaten mit abendländischen Staaten, also denen der respublica christiana ${ }^{75}$, im Vordergrund. Aussagen dazu finden sich dementsprechend im sog. Islamischen Völkerrecht. Den vorangegangenen Abschnitt zusammenzufassend ist festzuhalten:

1) Die Rechtsfigur des Dschihad verhindert die Anerkennung nichtmuslimischer Staaten als gleichberechtigte Partner.

2) Nichtmuslimischen Staaten wird Rechtssubjektivität nur im Bereich des amān auf Zeit zugesprochen.

3) Das Islamische Völkerrecht ist eine zeitlich und räumlich begrenzte Rechtsordnung, deren Subjekte die vertragsschließenden Staaten sind, wenn ein rechtsschöpfender Vertrag auf der Grundlage der $m u w \bar{a} d a^{c} a$ abgeschlossen wird. ${ }^{76}$

Somit war nach islamischem Recht die sog. Piraterie der Barbareskenstaaten durchaus zulässig auf der Grundlage des Dschihad. Im folgenden bleibt das Aufeinandertreffen des islamischen Rechts mit dem in Europa gültigen, christlich geprägten Recht, zu betrachten.

\section{Die christlich-abendländische Völkerrechtsgemeinschaft und die Muslime}

Die lange Zeit auf christliche Herrscher und Staaten zugeschnittene Völkerrechtsgemeinschaft wurde ab Mitte des 19. Jahrhunderts auch auf nichtchristliche Staaten ausgedehnt. ${ }^{77}$ Beziehungen zwischen Staaten unterschiedlicher religiöser Ausrichtung bestanden naturgemäß jedoch schon viel früher:

"In fact it would be wrong to suppose that there was not already in the sixteenth century a form of international law with its own conventions and some binding force. Islam and Christendom exchanged ambassadors, signed treaties and often respected their clauses. Insofar as the entire Mediterranean was an arena of constant conflict between two adjacent and warring civilizations, war was a permanent reality, excusing and justifying piracy; to justify it was to assimilate it to the neighbouring and in its way respectable category of privateering.",78

$\mathrm{Zu}$ der Bedeutung der bellum-iustum-Lehre, der respublica christiana sowie der Herausbildung des Völkerrechts s. J. Mössner, a.a.O., S. 36-55. Ausführlich hierzu bei H. Krüger, a.a.O., S. 33.

Siehe z.B. H. Krüger, a.a.O., S. 135, und K.-H. Ziegler, Zur Entwicklung von Kriegsrecht und Kriegsverhütung im Völkerrecht des 19. und frühen 20. Jahrhunderts, Archiv des Völkerrechts (2004), S. $271 \mathrm{f}$. 
Bis 1856 - bis zur Pariser Seerechts-Deklaration - war es im europäischen Rechtskreis erlaubt, dass ein Staat Privaten Kaperbriefe erteilte. Dabei ist die Einschätzung hinsichtlich Kapertum oder Piraterie nicht abhängig von der Frage, ob die Barbareskenstaaten eigenständige Staaten gewesen sind. Denn die Vorstellung vom Staat als dem alleinigen Kriegsherrn, der sein Kriegsführungsrecht ausnahmsweise auch Privaten übertragen kann, ist das Ergebnis der speziellen europäischen Entwicklung am Ende des Mittelalters. Sie beruht auf dem Gedanken des bellum iustum. ${ }^{79}$ Dem Islam ist die Vorstellung fremd, das Kriegsführungsrecht sei Vorrecht des Staates. Insbesondere im Rahmen des Dschihads sind alle Muslime zum Kampf gegen die Nichtmuslime aufgerufen. ${ }^{80}$ Im Vordergrund steht also vielmehr die Frage, ob die Barbareskenstaaten aus europäischer Sicht als Völkerrechtspersönlichkeiten galten oder nicht.

Zwar kann eine Untersuchung über völkerrechtliche Aspekte mit dem Ziel der Vergegenwärtigung historischer Sachverhalte heutige Begriffsinhalte zugrundelegen, doch lehnt der Völkerrechtler Mössner diesen Gedanken in Hinblick auf die Barbareskenstaaten zu Recht ab, da dieses Problem nicht nach dem Maßstab heutiger Theorien beantwortet werden könne. ${ }^{81}$ Mössner geht in seiner Dissertation zum Thema der Völkerrechtspersönlichkeit der Barbareskenstaaten den westlichen Vorstellungen über die Subjekte des Völkerrechts nach und stellt fest, dass Erörterungen über den Begriff der Völkerrechtspersönlichkeit im allgemeinen bis 1840 unbekannt waren. Der Begriff der Völkerrechtspersönlichkeit als einer "natürlichen" Eigenschaft der Staaten war das Ergebnis einer speziellen europäischen Geschichte. Auf außerhalb Europas stehende Staaten ist er deswegen nur dann anzuwenden, wenn diese eine ähnliche Geschichte aufweisen können - anderenfalls kann eine Übertragung von Vorstellungen über den Staat von einem Rechtskreis in den anderen der grundsätzlich verschiedenen Struktur nicht gerecht werden. Ein sachgerechtes Urteil kann dann zwangsläufig nicht erfolgen. ${ }^{82}$ Allerdings wurde die mittelbare Anerkennung der Völkerrechtspersönlichkeit durch den Abschluss von Verträgen erreicht, wie z.B. den Verträgen zwischen den Barbareskenstaaten und insbesondere den Staaten, die das Mittelmeer für die Seefahrt nutzten. Diese Verträge wurden in der Regel strikt eingehalten und ermöglichten in einem beschränkten Umfang den friedlichen Verkehr auf dem Mittelmeer. 83

Zum Abschluss solcher Verträge mit konstitutiver Wirkung konnte es im Einklang mit den islamischen Rechtsvorschriften kommen: "Da im Bereich der muwada'ah, des rechtschöp-

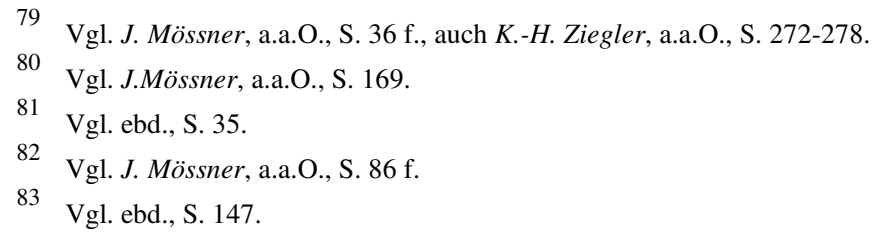


fenden Vertrages, sich zwei Verbände gegenüberstehen, die nach völkerrechtlichen Regeln die Voraussetzungen von Völkerrechtssubjekten erfüllen, ist es gerechtfertigt, das islamische Völkerrecht als temporär geltendes Völkerrecht zu bezeichnen." ${ }^{84}$

Nach älteren europäischen Rechtsansichten galt folgendes: Es handelte sich nicht um Seeräuberei, sondern um Kaperfahrten, die "im Staatsauftrag" erfolgten. Die Schiffsbesatzungen nahmen außerhalb eines völkerrechtlich anerkannten Krieges fremde Schiffe oder Schiffsladungen weg und wurden oft den Piraten gleichgestellt.

Nach dem neueren Modell der Völkerrechtstypen von Mössner ergeben sich Rechte und Pflichten nur im Rahmen der Vereinbarungen, und nur in diesem Maße können die Träger solcher Rechte und Pflichten als Völkerrechtspersönlichkeiten vorhanden sein. Im Völkerrechtstyp des sog. "Zwischen-Ordnungen-Rechts" begründet die Aufnahme vertraglicher Beziehungen die Anerkennung als Völkerrechtssubjekt und ist somit von konstitutiver Wirkung. Daraus resultierend ist für die Völkerrechtspersönlichkeit lediglich relevant, ob und wie diese als Träger von völkerrechtlichen Rechten und Pflichten in Erscheinung treten. Darauf Bezug nehmend ist bedeutungslos, ob sie die europäischen Merkmale eines Staates erfüllten. $^{85}$

\section{Ergebnis: Staatlich legitimierter Seeraub statt Piraterie}

Die Barbaresken waren keine Mitglieder der abendländisch-christlichen Völkerrechtsgemeinschaft, in der - trotz aller Divergenzen - eine gewisse Anerkennung von Regeln und Gesetzen bestand. Allerdings bahnte sich ab 1816 eine Annäherung an, als sie allmählich die Sklaverei und den Seeraub aufgaben.

Dessenungeachtet waren vielfältige Beziehungen zwischen den europäischen und den Barbareskenstaaten vorhanden. Diese bildeten ein eigenes Völkerrecht, das dem Völkerrechtstyp des Zwischen-Ordnungen-Rechts zugeschrieben werden kann. Nach diesem Recht waren die Barbaresken Völkerrechtspersönlichkeiten.

Der systematisch von den Barbareskenstaaten betriebene Seeraub ist nicht als Piraterie zu werten, sondern dem Dschihad zuzuordnen. Rechtlich gesehen gingen die Überfallenden auf Kaperfahrt: Sie brachten als Privatpersonen feindliche Handelsschiffe auf, was aufgrund des von ihrem kriegführenden Staat ausgestellten Kaperbriefes legitim war. Dass der Dschihad insbesondere durch die Teilnahme vieler Renegaten seinen religiösen Sinn häufig 
verlor und zu einem Raubzugskrieg wurde, wirkt sich wegen der engen Verknüpfung der verschiedenen Motive im Dschihad nicht auf die Beurteilung aus. ${ }^{86}$

\section{Ausblick: Verdrehung historischer Tatsachen und ihre Instrumentalisierung}

In den vergangenen Jahrzehnten war das Verhältnis zwischen Libyen und den USA von Spannungen geprägt. ${ }^{87}$ Die US-amerikanische Politik in diesem Zeitraum wird häufig als "Fortsetzung" der Libyen-Politik der Vereinigten Staaten im 18./19. Jahrhundert gewertet. Man mag diesen Umstand als ein Kontinuum beschreiben. Diese Annahme kann jedoch nicht zu der Beurteilung führen, dass es sich bei dem US-tripolitanischen Krieg (1801-05) um den "ersten Krieg der USA gegen den Terror" gehandelt habe. Zu diesem Ergebnis führen die Ausführungen über die Wertung von "Seeräuberei" sowohl gemäß dem europäischen als auch dem islamischen Rechtsdenken. Dennoch lassen sich Belege dafür finden, dass insbesondere US-Amerikaner im Rahmen der Auseinandersetzungen der USA mit dem Barbareskenstaat Tripolis Ende des 18. Jahrhunderts von einem "Ersten Krieg der USA gegen den Terror" sprechen. ${ }^{88}$ Diese Bezugnahme auf eine "Tradition der Konfrontation" ist nicht nur wenig dienlich und ungerechtfertigt, sie wird zudem auch unbesehen von insbesondere Journalisten rezipiert. ${ }^{89}$

Es soll an dieser Stelle keine ausführliche Darstellung der US-amerikanischen Mittelmeerpolitik dieser Zeitspanne geleistet werden. In den USA findet sich genügend ausführliches Material dazu. ${ }^{90}$ Auch in der Sekundärliteratur, die in Deutschland allerdings zu diesem Bereich kaum erhältlich ist, werden die Fakten in der Regel anders eingeordnet, als es die Verfechter von ähnlichen Thesen wie den folgenden tun: "Tripoli, Tunis, Algiers and Morocco were old hands at state-sponsored terrorism." "1 "Jefferson and his fighting sailors and Marines had freed America and Europe from The Terror.",92

86

87

88

89

90

91

92

Vgl. J. Mössner, a.a.O., S. 170.

Zu Einzelheiten s. A. Hinz, a.a.O., S. 190-200, S. 203-208.

Vgl. insbesondere J. Wheelan, Jefferson's War. America's First War on Terror 1801-1805, 2003.

Siehe z.B. Al-Qadِdāfî: al-umma șaqat bi-Amīrkā cadw aš-šucūu, in: Aš-šāhid (Nīqūsīyā), (1997)143, S. 13-17, [Al-Qadِdāfī: die Umma ist des Feindes der Völker Amerika überdrüssig]; W. Köhler, Von den Hallen Montezumas bis zu den Stränden von Tripolis, Frankfurter Allgemeine Zeitung, Nr. 198, 27.8.2001, S. 6; H. Sietz, Die Piraten von Tripolis. 200 Jahre "Krieg gegen den Terror" oder Wie Amerikas Soldaten 1801 erstmals in der islamischen Welt auftauchten, DIE ZEIT, Nr. 49, 29.11.2001, S. 104.

Vgl. Fn. 47.

J. Wheelan, a.a.O., S. 3.

Ebd., S. 368. 
Obwohl das Buch des Autors dieser Sentenzen auf umfangreichen Recherchen basiert, ordnet er die geschichtlichen Tatsachen jedoch - bewusst? - so an, dass die politischen Argumentationsmuster von heute für den Kampf gegen den Terrorismus auch den Entwicklungslinien vor mehr als zweihundert Jahren entsprechen sollen. Dabei missachtet der Verfasser den grundlegenden Unterschied, dass im Gegensatz zu heute mit den sog. "Terroristen" aus jener Zeit durchaus Abkommen bestanden haben, an denen zumindest aus juristischer Sicht - wie Mössner hinreichend nachgewiesen hat - nichts auszusetzen war. 